The microphotometric records of the lines $4046 \mathrm{~A}$. and $4077 \mathrm{~A}$. in the scattered spectrum and of the line $4046 \mathrm{~A}$. in the incident spectrum are reproduced in Fig. 1. The dotted part of the record in Fig. $1(a)$ would be obtained in absence of the wing. On measuring the relative intensities in different parts of

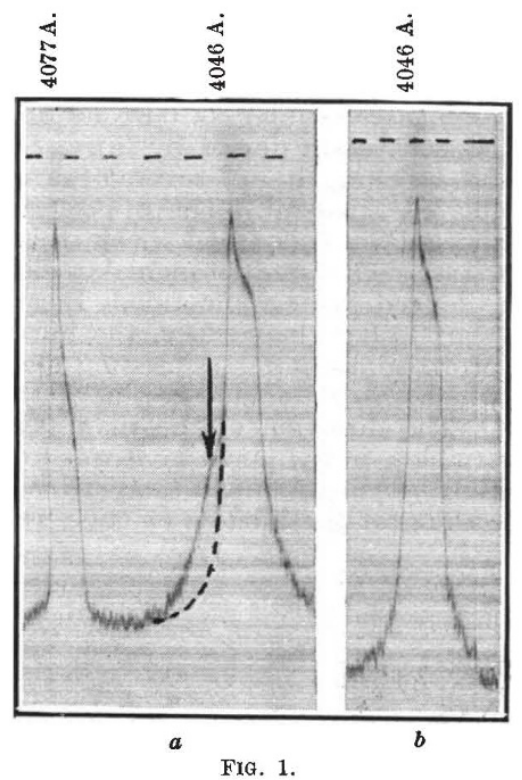

the wing, the curve reproduced in Fig. 2 is obtained. The approximate theoreticalcurvefor the benzene molecule at $210^{\circ} \mathrm{C}$. is shown by the broken line in Fig. 2. In view of the defects in the experimental arrangements, it can be concluded from the above results that there is fair agreement of the observed facts

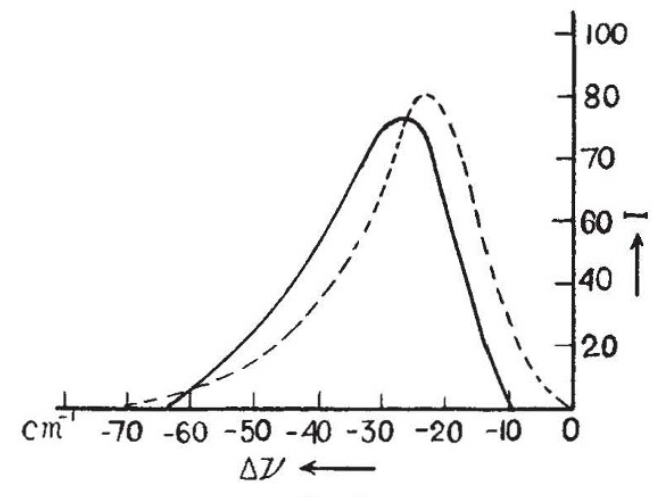

FIG. 2.

with the theory. Probably with a spectrograph having greater dispersion and with a denser picture, the agreement would be much better.

My thanks are due to Prof. D. M. Bose for his kind interest in the work.

Physics Department,

University College of Science,

92, Upper Circular Road, Calcutta.

Sept. 21.

'Z. Phys., 81, 209 ; 1933.

${ }^{2} Z$. Phys., 68, 782; 1931.

s Ind. J. Phys., 8, 437; 1934.
Bands at 4450 and $4180 \mathrm{~A}$. in the Spectra of the Night Sky and of the Aurora

IN the observation of the spectrum of the night sky or of the aurora by Vegard, Lord Rayleigh, Slipher, Sommer, Dufay, Ramanathan and others, it has already been reported that bands or lines appear in the regions near $4450 \mathrm{~A}$. and $4180 \mathrm{~A}$. These lines, which have been referred to by Lord Rayleigh as $X_{1}$ and $X_{2}$, were identified by Kaplan with the OII lines $4416 \cdot 97$ and $4169 \cdot 23$.

In the course of investigations of the band spectrum of nitrogen excited by very weak current under low pressure, I have observed three bands, which have heads at $4728 \cdot 5,4432 \cdot 3$ and $4165 \cdot 9 \mathrm{~A}$., respectively, in addition to other well-known bands. Of the three, the band at $4728.5 \mathrm{~A}$. is the weakest. Under low dispersion, each of these three bands has apparently four intensity maxima $(4744,4740,4736,4730$; $4448,4443,4439,4434 ; 4179,4175,4172,4167)$, and is little degraded towards the longer wavelength side. At ordinary temperature they are emitted only when the nitrogen gas is very pure; and the enhancement of their intensity relative to the second positive bands is observed with increased pressure of nitrogen, and with decreased density of the exciting current. Helium or neon gas exerts no appreciable effect when it was mixed with nitrogen, while the bands are almost quenched by the introduction of argon gas. At low temperature, especially at the temperature of liquid air, their intensity is markedly enhanced.

From the fact that the three bands are almost completely quenched when the temperature of nitrogen is high and when the persistence of vibration is brought out by the introduction of argon, and, therefore, the average vibrational and rotational energies of the normal nitrogen molecules are ab. normally high, it is concluded that the upper state of emission is very unstable.

Taking into consideration the wave-length regions where they appear, together with the exciting conditions, it seems that there is a possibility of the identification of the Rayleigh bands $X_{1}$ and $X_{2}$ in the spectra of the night sky, and perhaps also of the aurora, as the Goldstein bands which I have described above.

Physical Institute,

H. HAMAda.

Sendai, Japan.

Oct. 9.

\section{Nuclear Spin of Iodine}

IN an earlier report ${ }^{1}$ on the fine structures in the arc spectra of bromine and iodine, a tentative value of $\frac{9}{2}$ was proposed for the nuclear spin of iodine. The iodine arc spectrum was excited by a high-frequency electrodeless discharge, and as the structures were small and the individual components broad, it was pointed out that the spin value, which was based largely on one line, was not reliable because of imperfect resolution. It was definitely shown then that $I$ was certainly $\geqslant \frac{5}{2}$.

I have now succeeded in exciting both the arc and spark spectra of iodine in a cooled hollow cathode discharge, and the resulting lines are considerably sharper than in the previous source. The fine structures in the spark spectrum, being on a much larger scale, are sufficiently resolved in some lines to enable an unambiguous spin value to be determined. Some fine 